\title{
Long telomeres protect against age-dependent cardiac disease caused by NOTCH1 haploinsufficiency
}

\author{
Christina V. Theodoris, ${ }^{1,2,3}$ Foteini Mourkioti, ${ }^{4,5}$ Yu Huang, ${ }^{1,2}$ Sanjeev S. Ranade, ${ }^{1,2}$ Lei Liu, ${ }^{1,2}$ Helen M. Blau, ${ }^{4}$ \\ and Deepak Srivastava $a^{1,2,3,6}$
}

\begin{abstract}
${ }^{1}$ Cladstone Institute of Cardiovascular Disease and ${ }^{2}$ Roddenberry Center for Stem Cell Biology and Medicine, Cladstone Institutes, San Francisco, California, USA. ${ }^{3}$ Program in Developmental and Stem Cell Biology, UCSF, San Francisco, California, USA. ${ }^{4}$ Baxter Laboratory for Stem Cell Biology, Department of Microbiology and Immunology, Institute for Stem Cell Biology and Regenerative Medicine, Stanford University School of Medicine, Stanford, California, USA. ${ }^{5}$ Department of Orthopaedic Surgery, Department of Cell and Developmental Biology, Penn Institute for Regenerative Medicine, Perelman School of Medicine, University of Pennsylvania, Philadelphia, Pennsylvania, USA. ${ }^{6}$ Department of Pediatrics, Department of Biochemistry and Biophysics, UCSF, San Francisco, California, USA
\end{abstract}

\begin{abstract}
Diseases caused by gene haploinsufficiency in humans commonly lack a phenotype in mice that are heterozygous for the orthologous factor, impeding the study of complex phenotypes and critically limiting the discovery of therapeutics. Laboratory mice have longer telomeres relative to humans, potentially protecting against age-related disease caused by haploinsufficiency. Here, we demonstrate that telomere shortening in NOTCH1-haploinsufficient mice is sufficient to elicit age-dependent cardiovascular disease involving premature calcification of the aortic valve, a phenotype that closely mimics human disease caused by NOTCH1 haploinsufficiency. Furthermore, progressive telomere shortening correlated with severity of disease, causing cardiac valve and septal disease in the neonate that was similar to the range of valve disease observed within human families. Genes that were dysregulated due to NOTCH1 haploinsufficiency in mice with shortened telomeres were concordant with proosteoblast and proinflammatory gene network alterations in human NOTCH1 heterozygous endothelial cells. These dysregulated genes were enriched for telomere-contacting promoters, suggesting a potential mechanism for telomere-dependent regulation of homeostatic gene expression. These findings reveal a critical role for telomere length in a mouse model of age-dependent human disease and provide an in vivo model in which to test therapeutic candidates targeting the progression of aortic valve disease.
\end{abstract}

\section{Introduction}

Use of animal models to investigate age-dependent diseases in humans typically requires long-term aging. Furthermore, human diseases caused by genetic haploinsufficiency often fail to be modeled at any age in mice. Telomere shortening is thought to contribute to age-related diseases in humans, but this has yet to be demonstrated experimentally in an in vivo model. Many human diseases of homozygosity are recapitulated in mice, though in some cases, telomere shortening in mice homozygous for human disease mutations has revealed disease-related phenotypes $(1,2)$. It has yet to be demonstrated whether long telomeres in mice are protective against diseases of haploinsufficiency, which are frequently associated with age-related decline. Telomeres in lymphocytes of patients with calcific aortic valve disease (CAVD) are shortened, but whether telomere shortening is a causative factor is unknown (3). The incidence of CAVD increases with age, resulting in over 100,000 valve replacements for aortic valve stenosis (AS) (narrowing) annually in the United States. Fifty percent of individuals with CAVD also have coronary, aortic, or carotid artery calcification and therefore are at increased risk for myocardial infarctions and strokes.

Although CAVD often occurs in those with typical 3-leaflet aortic valves (AVs), a congenital anomaly of the AV in which the

Conflict of interest: The authors have declared that no conflict of interest exists.

Submitted: August 25, 2016; Accepted: January 31, 2017.

Reference information: J Clin Invest. 2017;127(5):1683-1688.

https://doi.org/10.1172/JCI90338. valve develops into 2 , rather than 3 , distinct leaflets (bicuspid aortic valve [BAV]) markedly raises the risk for CAVD. Approximately one-third of individuals with BAV, which occurs in $1 \%-2 \%$ of the population, will develop CAVD that typically has an earlier onset than usual. Congenital defects of the AV have high phenotypic variability, ranging from asymptomatic disease that becomes apparent later in life upon calcification to neonatal presentation with a thickened $\mathrm{AV}$ requiring immediate intervention. The most severe form of AS in the fetus can cause hypoplastic left heart syndrome, and all forms of AS are thought to be genetically linked. Humans with heterozygous NOTCH1 ( $h N 1)$ mutations, the only known genetic cause of AV disease, exhibit the full spectrum of this phenotypic variability (4-7). However, mice heterozygous for N1 have normal AVs (8), limiting further investigation in vivo.

\section{Results and Discussion}

To examine the role of telomere length in valve disease, we generated successive generations of $\mathrm{N1}^{+/-}$mice lacking telomerase activity by crossing C57BL/6 mice heterozygous for $N 1$ to those lacking the telomerase RNA component Terc (mTR) (9), as described in Supplemental Figure 1A (supplemental material available online with this article; https://doi.org/10.1172/ JCI90338DS1). Analyzing mice lacking telomerase activity over successive generations $\left(m \mathrm{TR}^{-/-}\right.$generation $1-3$, referred to as $\left.m T R^{G 1}-m T R^{G 3}\right)$ allowed the study of $N 1^{+/-}$mice with progressively shorter telomere lengths to determine the dose-dependent effect of telomere shortening on valve disease. 
A

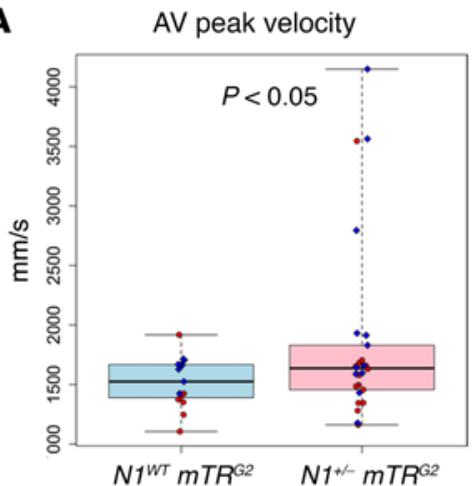

C

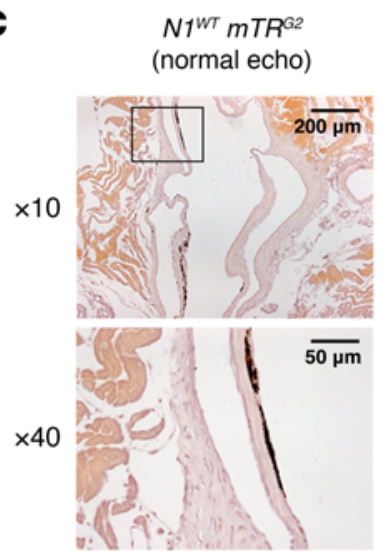

B

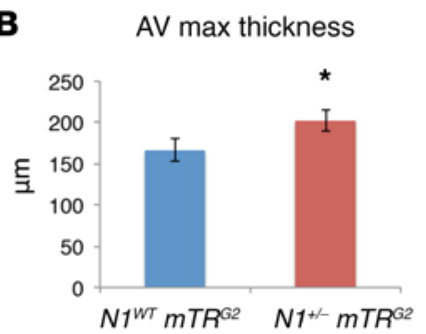

D

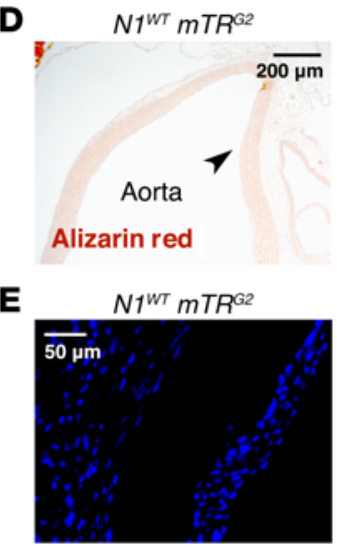

RUNX2 : DAPI

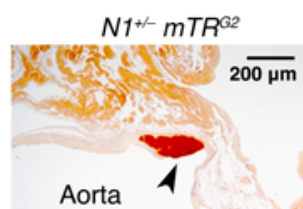

Alizarin red

$N 1^{+\infty} m T R^{G 2}$

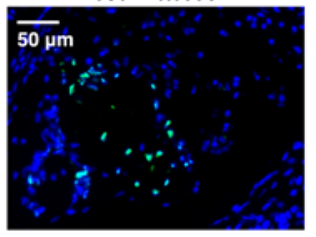

RUNX2 : DAPI

* $N 1^{+/-} m T R^{\mathrm{G} 2}$ (normal echo)

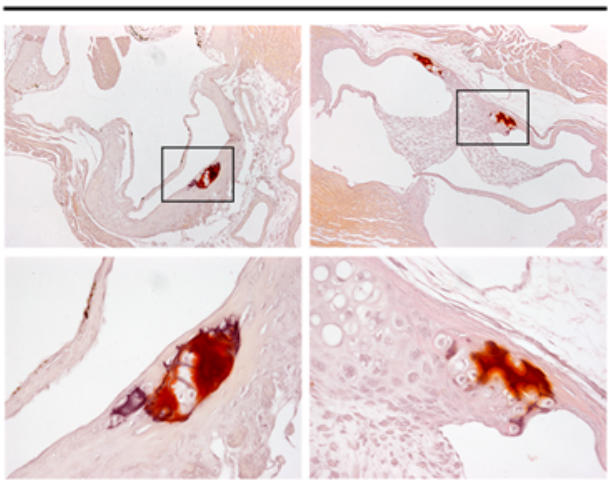

(AS on echo)

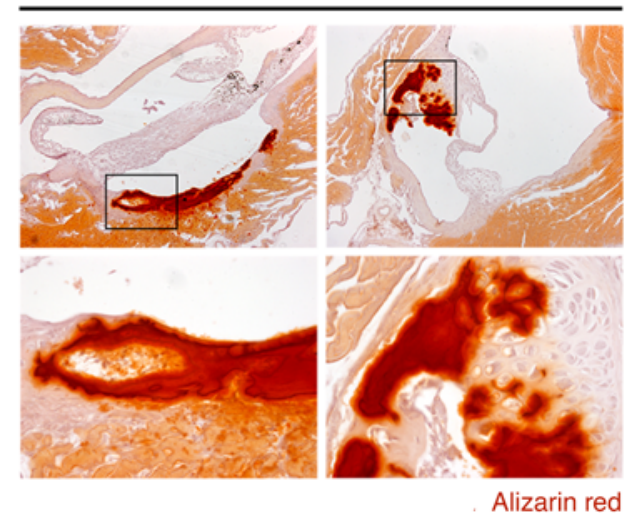

Figure 1. Telomere shortening elicits age-dependent AV calcification in $\mathbf{N 1 ^ { + / - }}$ mice. (A) AV peak velocity by echocardiography $\left(N 7^{w T} m T R^{G 2}, n=15 ; N 7^{+-}\right.$ $\left.m T R^{C 2}, n=26\right)$. Boxes show interquartile range (IQR); whiskers indicate range; lines indicate median. Red, female; blue, male. ${ }^{*} P<0.05,1$-sided $t$ test. (B) Mean AV leaflet maximum thickness ( $\left.N 1^{W T} m T R^{C 2}, n=14 ; N 1^{+/-} m T R^{C 2}, n=22\right)$. Data are shown as mean $\pm \mathrm{SEM}$. ${ }^{*} P<0.05,1$-sided $t$ test. (C) Alizarin staining (red) of calcification in AVs (N1 ${ }^{W T} m T R^{C 2}, 0 \%$ Alizarin positive, $n=16 ; N 1^{+-} m T R^{C 2}$ with normal echocardiograms, 30\% Alizarin positive, $n=17$; $\mathrm{N1}^{+-} m T R^{\mathrm{C2}}$ with AS by echocardiography, $100 \%$ Alizarin positive, $\left.n=4\right) .{ }^{*} P<0.05, \chi^{2}$ test. Boxes in upper row indicate region magnified in corresponding images in lower row. (D) Alizarin staining of calcification in aorta. Arrowheads indicate aortic wall. (E) RUNX2 and DAPI immunohistochemistry of AVs. $\mathrm{N1}^{+/-} \mathrm{mTR}^{\mathrm{G} 2} \mathrm{RUNX2}$-stained section is adjacent to bottom right Alizarin-stained section in $\mathbf{C}$.

We tested whether telomere shortening elicited the human CAVD phenotype in $\mathrm{N1}^{+/-}$mice by echocardiography. $\mathrm{N1}^{+/-} m \mathrm{mR}^{W T}$ mice had no abnormal echocardiographic findings of valve stenosis as 2-month-old adults $(n=6)$. At the same age, heart rate, left ventricular (LV) wall thickness, and ejection fraction were not significantly altered in $\mathrm{N1}^{+/-} m T R^{\mathrm{G} 2}$ mice (Supplemental Figure 1, B-D). However, in a bimodal distribution, a subset of $\mathrm{N1}^{+/-} m T R^{G 2}$ mice exhibited significantly decreased AV annulus diameter and increased AV peak velocity of blood flow and peak gradient of pressure across the AV, indicative of AS (Figure 1A and Supplemental Figure 1, E and F). Furthermore, leaflet thickening and defective closure of the AVs were apparent on histology and echocardiography (Figure 1B, and Supplemental Videos 1 and 2). The AVs were tricuspid, demonstrating that AS can occur even in the absence of bicuspid leaflets, as in humans and in families with N1 haploinsufficiency (4). $\mathrm{N1}^{+/-} m T R^{G 2}$ mice with the most narrowed $\mathrm{AV}$ diameters had disproportionately increased ascending aortic diameters, recapitulating the clinical phenotype of aortic dilation often observed in conjunction with AV disease and suggesting a genetic link between the two (Supplemental Figure $1, \mathrm{G}$ and $\mathrm{H}$ ).
Histologic examination of $N 1^{W T} m T R^{G 2}$ mice at 2 months of age did not reveal any evidence of calcification, marked by Alizarin staining (Figure 1C, $n=16$ ). In contrast, $100 \%$ of AVs examined from $\mathrm{N1}^{+/-} m T R^{G 2}$ mice with AS by echocardiography were positive for Alizarin staining (Figure 1C, $n=4$ ). The calcification was extensive, in many cases encompassing an entire leaflet of the $\mathrm{AV}$ and the aortic root and in some cases extending to the aortic vasculature (Figure 1D). Calcific regions showed coincident expression of the osteoblast transcriptional regulator RUNX2 (10), indicating an osteogenic switch (Figure 1E). 30\% of $\mathrm{N1}^{+/-} m \mathrm{mR}^{\mathrm{G} 2}$ mice with normal echocardiography showed calcification by Alizarin staining by 2 months of age, indicating variable disease progression (Figure 1C, $n=17)$. Overall, $43 \%$ of $\mathrm{N1}^{+-}-m T R^{G 2}$ mice had $\mathrm{AV}$ calcification, with $44 \%$ of those having progressed to AS by echocardiography $(n=21)$.

A subset of $\mathrm{N1}^{+-} m \mathrm{mR}^{\mathrm{G2}}$ mice also showed increased pulmonary valve (PV) peak velocity and peak pressure gradient of blood flow across the valve, indicative of PV stenosis (PS), compared with $N 1^{W T} m T R^{G 2}$ mice (Supplemental Figure 2, A and B). Mice with PS also had right ventricular (RV) hypertrophy secondary to the PV narrowing (Supplemental Figure 2, C and D). 100\% of mice with 
A

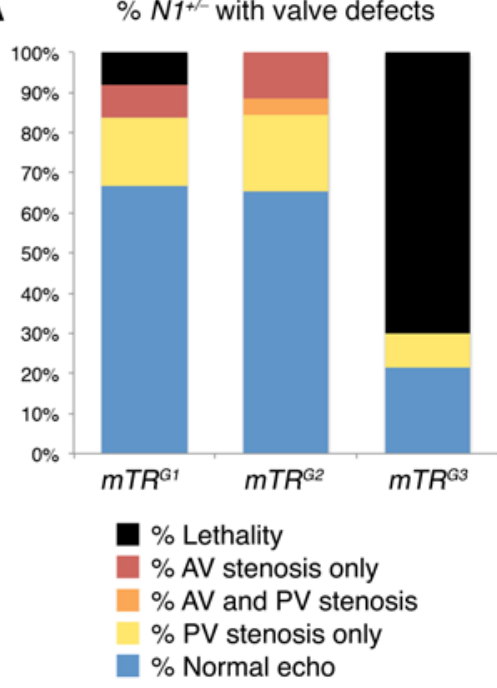

D

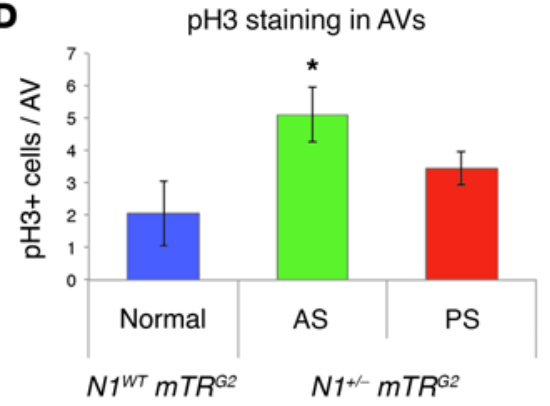

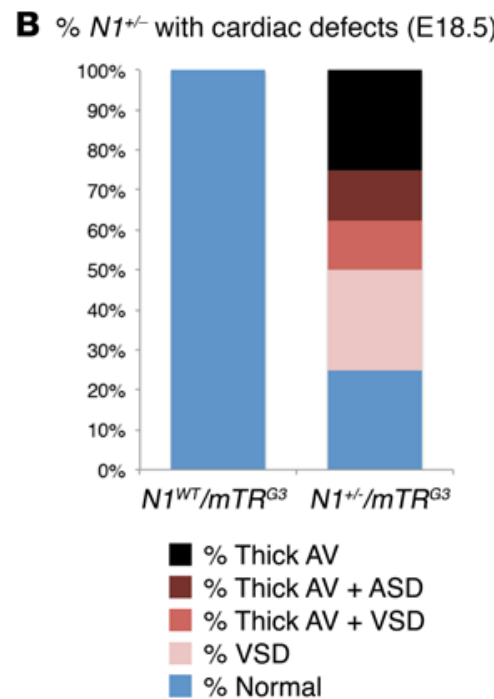

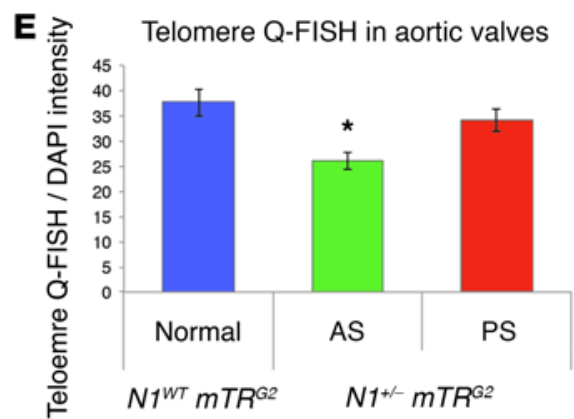

C

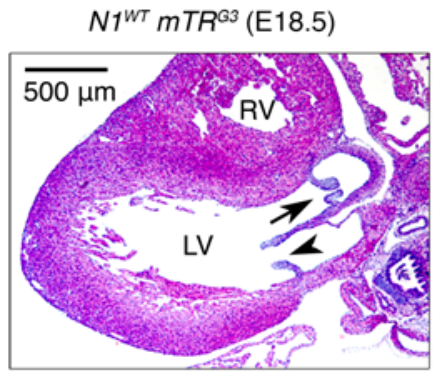

$N 1^{+/} m T R^{G 3}(E 18.5)$
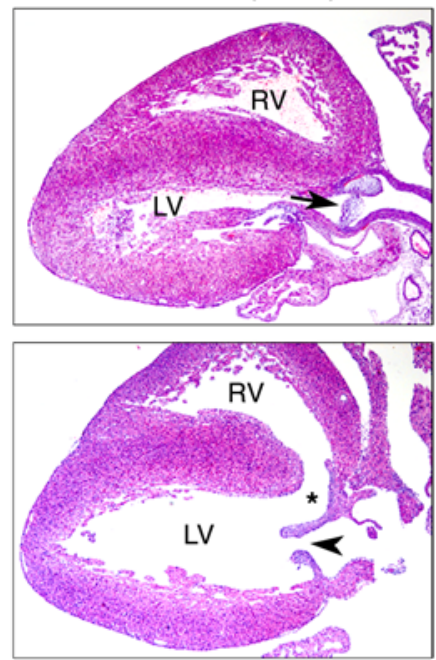

Figure 2. Disease severity in Notch1 mutants inversely correlates with telomere length. (A) Incidence of AS, PS, or lethality in $\mathrm{N1}^{+/-}$mice from successive mTR generations $\left(N 1^{+/} m T R^{G 1}, n=11 ; N 1^{+/-} m T R^{C 2}, n=26 ; N 1^{+/-} m T R^{C 3}, n=7\right)$. (B) Incidence of thick AV, ventricular septal defect (VSD), or atrial septal defect (ASD) in $m T R^{C 3}$ E18.5 embryos ( $N 7^{W T} m T R^{C 3}, n=13 ; N 1^{+-} m T R^{C 3}, n=8$ ). (C) Representative cross section at E18.5 of $N 7^{W T} m T R^{C 3}$ heart with normal anatomy (top) $(n=13), N 1^{+/-} m T R^{C 3}$ heart with AV leaflet thickening (middle) $(n=5)$, or $N 1^{+/-} m T R^{G 3}$ heart with ventricular septal defect (bottom, asterisk) ( $n=3$ ). Arrows indicate AVs. Arrowheads indicate mitral valves. (D) Quantification of pH3 staining for proliferation in adult $N 1^{W T} m T R^{C 2}(n=4)$ and $N 1^{+-} m T R^{C 2}(A S$ by echocardiography $n=5$, PS by echocardiography $n=5$ ) AVs. ${ }^{*} P<0.05$, 1-sided $t$ test, Benjamini-Hochberg correction. (E) Quantification of Q-FISH staining for telomere length in adult $N 7^{W T} m T R^{G 2}$ (hearts, 3; nuclei, 98) and $N 1^{+/-} m T R^{G 2}$ (AS by echocardiography hearts, 3; nuclei, 117; PS by echocardiography hearts, 2; nuclei, 78) AVs. ${ }^{*} P<0.05$, 1-sided $t$ test comparing individual nuclei. (D and E) Data are shown as mean \pm SEM.

PS by echocardiography showed thickened PV leaflets histologically, but lacked calcification (Supplemental Figure 2E, $n=4$ ). $\mathrm{N1}^{+/}$ $m T R^{G 2}$ mice that did not have PS by echocardiography had leaflets of intermediate thickness, with a total $33 \%$ of all $\mathrm{N1}^{+/}-m T R^{\mathrm{G} 2}$ mice exhibiting PV thickness more than 2 SD greater than that seen in $N 1^{W T} m T R^{G 2}$ mice (Supplemental Figure 2F, $n=24$ ). Interestingly, heterozygous mutations in N1 or its ligand JAG1 can also cause human PS or mitral valve stenosis, likely reflective of N1's regulation of endocardial cushions that contribute to cardiac valve development $(4,7,11,12)$. In total, $31 \%$ of $\mathrm{N1}^{+/-} m T R^{G 2}$ mice exhibited an echocardiographic defect in either of the semilunar (aortic or pulmonary) valves (only 1 mouse exhibited defects in both valves), while $55 \%$ exhibited a defect by histology at 2 months of age.

Although adult $N 1^{+/-} m T R^{\mathrm{G} 2}$ mice developed valve disease, neonatal $\mathrm{N1}^{+/-} m T R^{\mathrm{G} 2}$ mice showed no defects by echocardiography, indicating that the valve disease progresses in an age-dependent fashion ( $P<0.05$ by Barnard's test, neonatal, $n=11$; adult, $n=26)$. While AV defects were observed in the first generation, the severity of $\mathrm{AV}$ defects increased between the first and second generations of mice lacking $m T R$. By the third generation, we observed approx- imately $70 \%$ lethality by weaning age (Figure 2A and Supplemental Figure $3 \mathrm{~A}$ ), and $75 \%$ of $\mathrm{N1}^{+/-} m \mathrm{TR}^{\mathrm{G} 3}$ animals at $\mathrm{E} 18.5$ had thickened AV leaflets and/or septal defects, explaining the early lethality (Figure 2, B and C, and Supplemental Figure 3B). Because a spectrum of $\mathrm{AV}$ disease, ranging from adult onset CAVD to newborn AS, is often observed within families, it is intriguing to consider telomere length as a contributor to phenotypic variability.

Since N1 is thought to stabilize endothelial cells (ECs), preventing their proliferation (13), we investigated whether increased proliferation in the valves of $\mathrm{N1}^{+/-} m T R^{\mathrm{G} 2}$ mice might result in further telomere shortening. Phospho-histone H3 (pH3) immunostaining revealed increased proliferation specifically in the valve affected by stenosis in N1-haploinsufficient mice, with no difference in apoptosis by TUNEL staining (Figure 2D and Supplemental Figure $3 \mathrm{C}-\mathrm{F}$ ). $\mathrm{N1}^{+/-} m T R^{\mathrm{G} 2}$ mice with AS had increased proliferation in the AV compared with $N 1^{W T} m T R^{G 2}$ mice and $N 1^{+/-} m T R^{G 2}$ mice with PS (Figure 2D). Conversely, mice with PS had increased proliferation in the PV compared with $N 1^{W T} m T R^{G 2}$ mice and $N 1^{+/-}$ $m T R^{G 2}$ mice with AS (Supplemental Figure 2F). Correspondingly, $\mathrm{N1}^{+/-} m T R^{G 2}$ mice with AS showed significantly reduced telomeres 
A

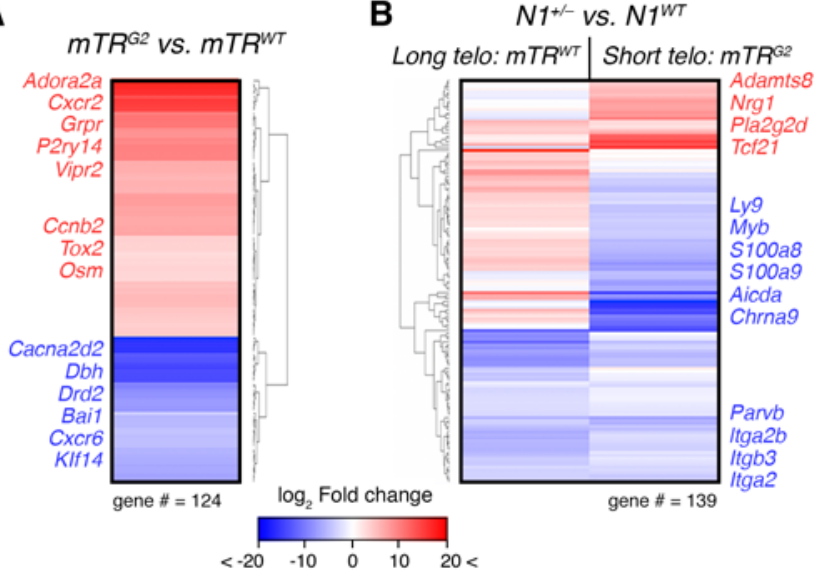

C

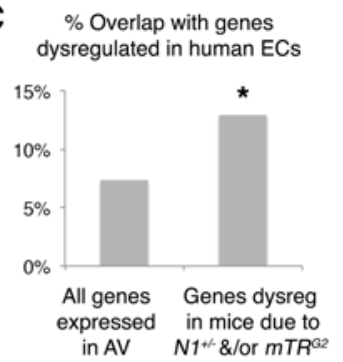

D

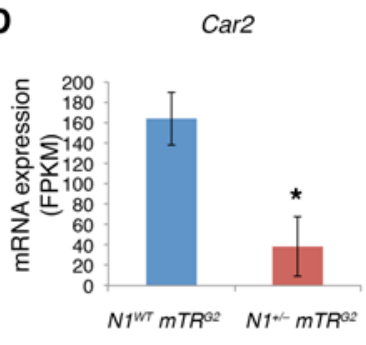

\section{F Fold enrichment of telomere-contacting}

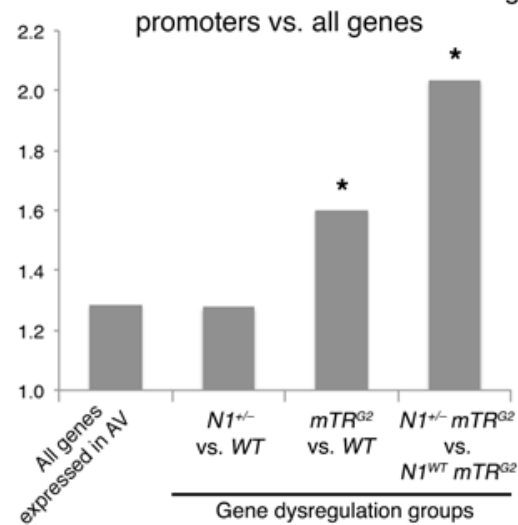

G

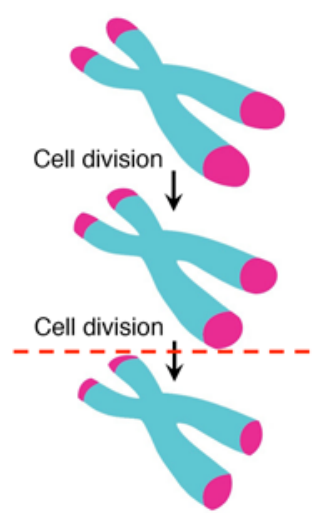

E

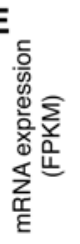

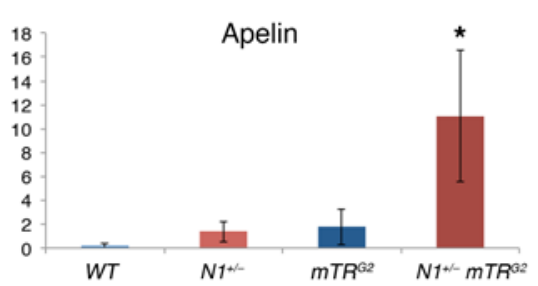

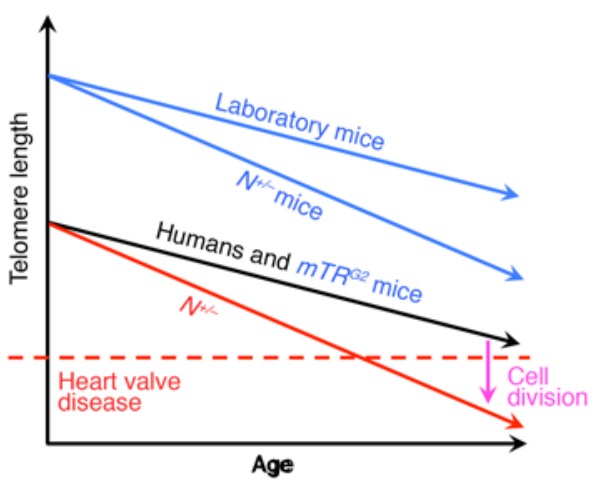

Figure 3. Genes dysregulated due to telomere shortening are enriched for telomere-contacted promoters. (A) mRNA expression by RNA-seq of dysregu-

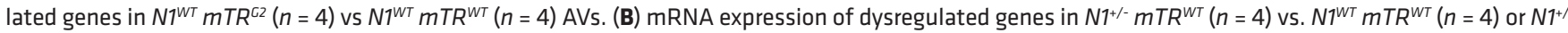
$m T^{G 2}(n=4)$ vs N1 ${ }^{W T} m T R^{C 2}$ AVs $(n=3)$. Telo, telomere. (C) Percentage of overlap of indicated murine gene groups with genes dysregulated (dysreg) due to N1 heterozygosity in human iPSC-derived ECs (16). ${ }^{*} P<0.05, \chi^{2}$ test. ( $\mathbf{D}$ and $\mathbf{E}$ ) Mean mRNA expression of indicated genes in WT $(n=4), N 1^{+-}(n=4), m T R^{\mathrm{C2}}$ $(n=3)$, and $N 1^{+/-} m T R^{C 2}(n=4)$ AVs. Data are shown as mean \pm SEM. ${ }^{*}$ Significant by negative binomial test, false discovery rate (FDR) correction of $10 \%$. FPKM, fragments per kilobase million. (F) Fold enrichment of telomere-contacting promoters in stated gene groups compared with all genes. ${ }^{*} P<0.05$, hypergeometric test, Benjamini-Hochberg correction. (G) Model of telomere length and relationship to disease onset. Left, telomere length shortens with each cell division. Right, telomere length varies at baseline by organism and mTR genotype and progressively shortens with aging as cells divide over time. Dotted line, threshold after which heart valve disease may ensue. Increased cell division caused by N1 haploinsufficiency accelerates telomere shortening rate, eliciting the heart valve disease phenotype in $\mathrm{N1}^{+/-} \mathrm{mTR}^{\mathrm{C2}}$ mice.

specifically in their AVs compared with $N 1^{W T} m T R^{G 2}$ mice or $\mathrm{N1}^{+/-}$ $m T R^{G 2}$ mice without AS (Figure 2E and Supplemental Figure 3, $\mathrm{G}$ and $\mathrm{H}) . \mathrm{N1}^{+/-} m T R^{\mathrm{G} 2}$ mice with PS also had reduced telomeres specifically in their PVs compared with $N 1^{W T} m T R^{G 2}$ mice or $\mathrm{N1}^{+/-}$ $m T R^{G 2}$ mice without PS (Supplemental Figure 3, G and H). Thus, increased proliferation in diseased valves correlated with further telomere shortening in those valves, with telomere shortening below a quantitative FISH (Q-FISH) signal threshold of approximately 30 units segregating with the diseased valve.

To determine the genes dysregulated due to telomere shortening and how N1 haploinsufficiency differentially affected AVs in the context of the baseline telomere length, we performed RNA sequencing on dissected $\mathrm{AVs}$ from $\mathrm{WT}, \mathrm{N1}^{+/-}, \mathrm{mTR}^{\mathrm{G} 2}$, and $\mathrm{N1}^{+/-}$ $m T R^{G 2}$ mice. Telomere shortening alone $\left(N 1^{W T} m T R^{G 2}\right)$ resulted in dysregulation of genes enriched in gene ontology terms related to GPCRs and $\beta$ receptor response (Figure 3A and Supplemental Tables 1 and 2). GPCRs are one of the few known classes of pro- teins that serve as direct mechanosensors of hemodynamic shear stress in ECs (14). Given that laminar shear stress protects against atherosclerosis and CAVD (15), age-dependent telomere shortening may place AVs at greater risk by disrupting protective shearinduced GPCR signaling. Shear-induced N1 signaling also activates anticalcific gene networks in ECs (16-18), raising the possibility that N1 haploinsufficiency and dysregulated GPCR signaling in telomere-shortened AVs may contribute to the accelerated age-dependent calcification in $\mathrm{N1}^{+/-}$individuals.

Indeed, N1 haploinsufficiency in mice with long telomeres $\left(\mathrm{N1}^{+/-} m T R^{W T}\right)$ downregulated genes involved in cell adhesion, including multiple integrins and actin-binding genes, including Parvb, which has been previously linked to coronary atherosclerosis (19) (Figure 3B). N1-haploinsufficient mice with shortened telomeres $\left(\mathrm{N1}^{+/-} m T R^{62}\right)$ also upregulated $T c f 21$, which promotes migration of profibrotic cells to atherosclerotic lesions in vivo (20), and $N r g 1$, which may contribute to proliferation within thickened 
valve leaflets (21) (Figure 3B). More broadly, gene dysregulation in $N 1^{+/-} m T R^{G 2}$ mice had significant overlap with genes perturbed in human $N 1^{+/-}$ECs (16-17), in which telomeres are naturally shorter, including those involved in proosteogenic and proinflammatory pathways (Figure 3C and Supplemental Figure 3I). For example, $\mathrm{N1}^{+/-} m T R^{\mathrm{G} 2} \mathrm{AVs}$ downregulated osteoclast factors such as Car2 (22), important for bone resorption, compared with $N 1^{W T} m T R^{G 2}$ AVs. Additionally, like human $\mathrm{N1}^{+/-}$ECs (16), $\mathrm{N1}^{+/-} m \mathrm{mR}^{\mathrm{G} 2} \mathrm{AVs}$ upregulated both Apelin and its receptor, which are therapeutic targets for atherosclerosis and upregulated in human coronary atherosclerotic plaques as well as calcified human AVs (23) (Figure 3, D and E, and Supplemental Figure 3J).

3D chromatin analysis suggests telomeres loop back to promoters in the genome and thereby affect gene expression (24). We examined the reported sites of telomere contacts genome-wide in human umbilical vein ECs (25) and found that genes dysregulated in telomere-shortened $\mathrm{mTR}^{\mathrm{G} 2}$ valves were enriched for telomere-contacting promoters compared with all genes (Figure 3F). Furthermore, genes dysregulated in $\mathrm{N1}^{+/-} \mathrm{mTR}^{G 2}$ valves, which have even further reduced telomere length compared with $N 1^{W T} m T R^{G 2}$ valves, were even more enriched for telomere-contacting promoters. Thus, genes dysregulated with decreasing telomere length were increasingly likely to be regulated by promoters associated with telomeres within the $3 \mathrm{D}$ chromatin structure in the nucleus, suggesting that disruption of this looping with telomere shortening may result in gene dysregulation responsible for age-related CAVD.

This study reveals that telomere shortening erodes the tolerance to haploinsufficiency in age-related disease and that shortening of long telomeres protective in mice can accurately recapitulate a human disease of haploinsufficiency. It is remarkable that despite the broad function of $\mathrm{N} 1$ in a myriad of tissues, telomere shortening in $N 1$ heterozygous mice resulted only in cardiovascular disease, precisely recapitulating the clinical phenotype observed in humans with N1 haploinsufficiency. Reduced dosage of N1 led to upregulation of cell cycle genes and increased cell proliferation in the valve, which may shorten telomeres over time past a critical threshold (Figure 3G). Calcification of the aortic vasculature, commonly observed in humans with CAVD in an age-dependent manner, was also elicited by telomere shortening. It is interesting that progressive shortening of telomeres not only increased severity of disease, but also mimicked the range of $\mathrm{AV}$ disease seen in humans, including neonatal onset. The mechanism by which telomere length affects aging or disease more broadly remains unclear, but the enrichment in genes contacted by telomere looping among those dysregulated in mouse $\mathrm{N1}^{+/-}$AVs with shortened telomeres suggests a potential mechanism for telomere-dependent regulation of homeostatic gene expression.

While we focused on heterozygosity of $N 1$, the majority of human diseases of haploinsufficiency lack a phenotype in mice heterozygous for the orthologous factor, similar to N1. In contrast, many human diseases of homozygosity are recapitulated in mice, although in the case of Duchenne muscular dystrophy, the mouse phenotype caused by homozygous mutations in $M d x$ was only fully manifested upon telomere shortening $(1,2)$. It will be interesting to determine whether telomere shortening represents a more general approach to modeling human disease in mice, particularly those diseases caused by dose-sensitive mutations. The model described here provides a unique opportunity for dissecting the mechanisms by which telomeres affect age-dependent disease and specifically provides an in vivo system for testing novel therapeutics for AV disease in adults and neonates.

\section{Methods}

Experimental details are in Supplemental Methods. All original RNA sequencing data were deposited in the NCBI's Gene Expression Omnibus (GEO GSE83963).

Study approval. Protocols were reviewed and approved by the UCSF Institutional Animal Care and Use Committee.

\section{Author contributions}

CVT designed and performed experiments and bioinformatic analyses. FM designed, performed, analyzed, and interpreted telomere Q-FISH experiments and edited the manuscript. YH performed echocardiography. SSR and YH performed E18.5 dissections. CVT, YH, SSR, and LL performed perfusion fixation, mouse breeding, and genotyping. HMB designed experiments and analyses, supervised telomere Q-FISH experiments and interpretation, and edited the manuscript. DS designed experiments and analyses and supervised the work. CVT and DS wrote the manuscript.

\section{Acknowledgments}

We are grateful to the Srivastava Lab and Gladstone/UCSF members for scientific discussions. We thank the Gladstone Histology, Genomics, and Bioinformatics Cores for technical expertise. We thank B. Taylor for editorial help and K.N. Ivey for critical comments. DS was supported by the L.K. Whittier and Roddenberry Foundations, the Younger Family Fund, the California Institute for Regenerative Medicine (CIRM, grant DISC2-08098), and the $\mathrm{NIH} /$ National Heart, Lung, and Blood Institute/National Center for Research Resources (U01-HL098179, U01-HL100406, C06-RR018928). HMB was supported by the Baxter Foundation and the NIH (AG044815, AG009521, NS089533, AR063963, AG020961). CVT was supported by the American Heart Association (AHA), Roddenberry, Winslow, and UCSF Discovery Fellowships, the UCSF Program in Developmental and Stem Cell Biology (NIH-T32HD007470), and the Medical Scientist Training Program (MSTP) (NIH-T32GM007618). FM was supported by the AHA (10SDG3510024) and the Perelman School of Medicine.

Address correspondence to: Deepak Srivastava, 1650 Owens Street, San Francisco, California 94158, USA. Phone: 415.734.2716; E-mail: dsrivastava@gladstone.ucsf.edu.
1. Sacco A, et al. Short telomeres and stem cell exhaustion model Duchenne muscular dystrophy in mdx/mTR mice. Cell. 2010;143(7):1059-1071.

2. Mourkioti F, et al. Role of telomere dysfunction in cardiac failure in Duchenne muscular dystrophy.
Nat Cell Biol. 2013;15(8):895-904.

3. Kurz DJ, et al. Degenerative aortic valve stenosis, but not coronary disease, is associated with shorter telomere length in the elderly. Arterioscler Thromb Vasc Biol. 2006;26(6):e114-e117.
4. Garg V, et al. Mutations in NOTCH1 cause aortic valve disease. Nature. 2005;437(7056):270-274

5. McKellar SH, Tester DJ, Yagubyan M, Majumdar R, Ackerman MJ, Sundt TM. Novel NOTCH1 mutations in patients with bicuspid aortic valve 
disease and thoracic aortic aneurysms. J Thorac Cardiovasc Surg. 2007;134(2):290-296.

6. Mohamed SA, et al. Novel missense mutations (p.T596M and p.P1797H) in NOTCH1 in patients with bicuspid aortic valve. Biochem Biophys Res Commun. 2006;345(4):1460-1465.

7. Kerstjens-Frederikse WS, et al. Cardiovascular malformations caused by NOTCH1 mutations do not keep left: data on 428 probands with left-sided CHD and their families. Genet Med. 2016;18(9):914-923.

8. Nus M, et al. Diet-induced aortic valve disease in mice haploinsufficient for the Notch pathway effector RBPJK/CSL. Arterioscler Thromb Vasc Biol. 2011;31(7):1580-1588.

9. Blasco MA, et al. Telomere shortening and tumor formation by mouse cells lacking telomerase RNA. Cell. 1997;91(1):25-34.

10. Ducy P, Zhang R, Geoffroy V, Ridall AL, Karsenty G. Osf2/Cbfa1: a transcriptional activator of osteoblast differentiation. Cell.1997;89(5):747-754.

11. Colliton RP, Bason L, Lu FM, Piccoli DA, Krantz ID, Spinner NB. Mutation analysis of Jagged1 (JAG1) in Alagille syndrome patients. Hum Mutat. 2001;17(2):151-152.

12. Eldadah ZA, et al. Familial tetralogy of Fallot caused by mutation in the jagged1 gene. Hum Mol Genet. 2001;10(2):163-169.
13. Liu ZJ, et al. Inhibition of endothelial cell proliferation by Notch 1 signaling is mediated by repressing MAPK and PI3K/Akt pathways and requires MAML1. FASEB J. 2006;20(7):1009-1011.

14. Chachisvilis M, Zhang YL, Frangos JA. G proteincoupled receptors sense fluid shear stress in endothelial cells. Proc Natl Acad Sci USA. 2006;103(42):15463-15468

15. Weinberg EJ, Mack PJ, Schoen FJ, García-Cardeña G, Kaazempur Mofrad MR. Hemodynamic environments from opposing sides of human aortic valve leaflets evoke distinct endothelial phenotypes in vitro. Cardiovasc Eng. 2010;10(1):5-11.

16. Theodoris CV, et al. Human disease modeling reveals integrated transcriptional and epigenetic mechanisms of NOTCH1 haploinsufficiency. Cell. 2015;160(6):1072-1086.

17. White MP, et al. NOTCH1 regulates matrix gla protein and calcification gene networks in human valve endothelium. J Mol Cell Cardiol. 2015;84:13-23.

18. Masumura T, Yamamoto K, Shimizu N, Obi S, Ando J. Shear stress increases expression of the arterial endothelial marker ephrinB2 in murine ES cells via the VEGF-Notch signaling pathways. Arterioscler Thromb Vasc Biol. 2009;29(12):2125-2131.

19. Verschuren JJ, et al. Pathway analysis using genome-wide association study data for coronary restenosis--a potential role for the PARVB gene. PLoS One. 2013;8(8):e70676.

20. Nurnberg ST, et al. Coronary Artery Disease Associated Transcription Factor TCF21 Regulates Smooth Muscle Precursor Cells That Contribute to the Fibrous Cap. PLoS Genet. 2015;11(5):e1005155.

21. Hedhli N, et al. Endothelial-derived neuregulin is an important mediator of ischaemia-induced angiogenesis and arteriogenesis. Cardiovasc Res. 2012;93(3):516-524.

22. Lehenkari P, Hentunen TA, Laitala-Leinonen T, Tuukkanen J, Väänänen HK. Carbonic anhydrase II plays a major role in osteoclast differentiation and bone resorption by effecting the steady state intracellular $\mathrm{pH}$ and Ca2+. Exp Cell Res. 1998;242(1):128-137.

23. Lv D, Li H, Chen L. Apelin and APJ, a novel critical factor and therapeutic target for atherosclerosis. Acta Biochim Biophys Sin (Shanghai). 2013;45(7):527-533.

24. Robin JD, et al. Telomere position effect: regulation of gene expression with progressive telomere shortening over long distances. Genes Dev. 2014;28(22):2464-2476.

25. Rao SS, et al. A 3D map of the human genome at kilobase resolution reveals principles of chromatin looping. Cell. 2014;159(7):1665-1680. 